CBP-810

\title{
Use of the Lorentz-Boosted Frame Transformation to Simulate Free-Electron Laser Amplifier Physics
}

\author{
W.M. Fawley and J.-L. Vay \\ Center for Beam Physics, Lawrence Berkeley National Laboratory, Berkeley, CA 94720
}

\begin{abstract}
.
Recently [1] it has been pointed out that numerical simulation of some systems containing charged particles with highly relativistic directed motion can by speeded up by orders of magnitude by choice of the proper Lorentz boosted frame. A particularly good example is that of short wavelength free-electron lasers (FELs) in which a high energy $\left(E_{0} \geq 250 \mathrm{MeV}\right)$ electron beam interacts with a static magnetic undulator. In the optimal boost frame with Lorentz factor $\gamma_{F}$, the red-shifted FEL radiation and blue shifted undulator have identical wavelengths and the number of required time-steps (presuming the Courant condition applies) decreases by a factor of $\gamma_{F}^{2}$ for fully electromagnetic simulation.

We have adapted the WARP code [2] to apply this method to several FEL problems including coherent spontaneous emission (CSE) from pre-bunched e-beams, and strong exponential gain in a single pass amplifier configuration. We discuss our results and compare with those from the "standard" FEL simulation approach which adopts the eikonal approximation for propagation of the radiation field.
\end{abstract}

Keywords: free-electron laser, electromagnetic simulation algorithms

PACS: $41.60 . \mathrm{Cr} 41.20 . \mathrm{Jb} \quad 41.60 .-\mathrm{m} 42.25 . \mathrm{Kb}$

\section{INTRODUCTION}

It is well known that in general, explicit, fully electromagnetic simulation will have its time step $\Delta t$ limited by the Courant condition corresponding to the numerical grid spacing and/or that necessary to achieve sufficient temporal resolution of the highest frequencies important to the physics of the particular situation. For problems in which a highly relativistic charged particle beam is present, the overall system time and/or length scale $L_{s i m}$ can be large and the ratio of scale lengths $L_{s i m} / c \Delta t$ can become enormous. Recently, Vay [1] pointed out that for some of these problems performing the simulation in a Lorentz-boosted frame offers potentially orders of magnitude speedup in computation time.

A natural candidate for boosted frame calculations is a short wavelength free-electron laser (FEL). Here a sample problem could have the resonant radiation wavelength $\lambda_{R}=10 \mathrm{~nm}$, an undulator wavelength $\lambda_{u}=25 \mathrm{~mm}$, and a system length $L_{s i m} \geq 10 \mathrm{~m}$. Performing this simulation in the laboratory frame requires $\sim 4 \times 10^{9}$ time steps or greater. Presuming a "moving window" type simulation centered about the electron beam of length $l_{b} \approx 100 \mu \mathrm{m}$ and radius $r_{b} \approx 50 \mu \mathrm{m}$, the number of grid points for an 2D axisymmetric (or slab) model exceeds $10^{8}$ (and likely 3-10 times greater to properly model transverse diffraction effects). 
Even today with massively parallel systems, doing just one fully electromagnetic simulation in the lab frame with these parameters would be an enormous undertaking, and not one that scales easily to multi-parameter investigations. Instead, realizing for most problems that the normalized gain bandwidth is small and that the radiation gain length $L_{G} \gg \lambda_{R}$, for a quarter century or more FEL simulationists have applied the eikonal approximation (also known as the slowly-varying envelope approximation). This changes the E\&M field equations from hyperbolic to parabolic (e.g., diffusive) in nature, thereby permitting $\Delta z$ and $\Delta t$ to approach $\sim L_{G} / 8$ and $\sim 0.1 \lambda_{R}^{2} / c \Delta \lambda_{B W}$, respectively. Numerically, this leads to the grid spacings $\Delta z$ and $\Delta r$ being of order centimeters and microns, respectively, rather than nanometers, resulting in the total number of grid points being $10^{6}$ or less and time steps being $10^{3}$ or less with an overall speedup of $10^{8}$ or greater over full electromagnetic simulation.

The natural' boosted frame for FEL computations is the so-called "ponderomotive" frame in which the e-beam longitudinal speed (when in the undulator) is zero on average. In this frame the red-shifted FEL resonant wavelength $\lambda_{R}^{\prime}=2 \gamma_{F} \lambda_{R}$ is equal to the blueshifted undulator wavelength $\lambda_{u}^{\prime}=\lambda_{u} / \gamma_{F}$. Here $\gamma_{F}^{2} \equiv \gamma_{0}^{2} /\left(1+a_{w}^{2}\right)$ with $a_{w}$ being the normalized, RMS undulator strength. The Lorentz transformation to the boosted frame shrinks the undulator by a factor $\gamma_{F}$ and increases the radiation wavelength by the same factor times two, resulting in an overall decrease of the needed number of time steps by a factor $\approx 2 \gamma_{F}^{2}$. Likewise, from the point of view of the Courant condition, the increase in $\Delta t$ permits (in general) a similar increase in the spatial grid zone size so that the savings in 2- and 3-D simulations can be immense. However, in cases where the electron beam is much longer than the so-called slippage length $\left(l_{\text {slip }} \equiv N_{u} \lambda_{R}\right)$ in the lab frame, this length exceeds the undulator length in the boosted frame and one factor of $\gamma_{F}$ is lost (i.e., the ratio of $l_{b} / \lambda_{R}$ remains constant independently of $\gamma_{F}$ ).

To study various standard FEL problems, we used the WARP simulation code [2] with its standard full E\&M solver in slab-mode geometry $(e . g ., x-z$ or $y-z)$. A special Python script implemented the linearly-polarized undulator fields in the boosted frame. For amplifier runs, a second script that initialized the input radiation at the upstream longitudinal boundary (in the boosted frame) also was applied. Special diagnostics were added which measured the forward-moving radiation intensity through a transverse plane fixed in the lab frame (e.g., at a fixed $z$ relative to the undulator entrance). In order to avoid requiring initialization of the $E$ - and $B$-fields associated with a beam pulse with a net current and charge, we "added" a positron beam with the exact same charge and current distribution at $t=0$ in the simulation; this doubles the effective current for radiation production. When these beams enter the transverse fields of the undulator, they are separated in the wiggle plane, creating an attractive (but unphysical) electric field. However, for optical and shorter wavelength FEL's operating in the Compton regime (e.g., $\left.I_{B} \leq 10 \mathrm{kA}\right)$ we expect these additional forces to be negligible in terms of radiation production (as was spot-checked by removing the faux positron beam).

We now present example results for some short wavelength test cases of stimulated emission from pre-bunched beams and a high-gain amplifier. Due to space limitations, a more detailed exposition will be given elsewhere. 


\title{
SIMULATIONS OF COHERENT STIMULATED EMISSION (CSE) FROM A PRE-BUNCHED BEAM
}

\author{
Emission by a Single Ultrashort Bunch
}

A number of simulations were done examining the emission from an ultrashort bunch $\left(l_{b} \ll \lambda_{R}\right)$ traveling through a moderate length undulator. Theoretically, one expects the total emission to scale as $Q_{b}^{2} N_{u} a_{w}^{2} /\left(1+a_{w}^{2}\right)$. This scaling was confirmed in the WARP runs in the boosted frame, as was the relative growth of harmonic emission as $a_{w}$ approached 1. As expected, even harmonic emission peaked off-axis; however, it was not exactly zero on-axis because of the presence of 2 nd and other even harmonic components in the undulator field associated with the ramp in strength at undulator entrance and exit. As $N_{u}$ increased, this on-axis, even harmonic emission became proportionately less and less relative to the fundamental and odd harmonics.

One advantage of an full E\&M code over an eikonal code is that it can study situations where there is broadband emission and/or conditions where the slowly-varying envelope approximation are no longer strongly valid. Such a case occurs in the entrance and exit ramp regions of the undulator where the local $B_{\text {peak }}$ (and thus the local value of $\lambda_{R}$ ) changes significantly over a few undulator periods or less. We studied the role of the ramp length for the flux from a single, low charge micropulse with optical phase extent $\pi / 4$ propagating in an undulator of total length $0.5 \mathrm{~m}$. With a functional matching ramp dependence of $B_{\perp}=B_{0}\left(z / L_{M}\right) \sin \left(2 \pi z / \lambda_{u}\right)$ for $0 \leq z \leq L_{M}$, we did a series of simulations varying $L_{M}$ from 2 to $8 \lambda_{u}$ with $\lambda_{u}=25 \mathrm{~mm}$. The other FEL parameters were $\lambda_{R}=200 \mathrm{~nm}, a_{w}=0.2, \gamma_{0}=255$, and $Q_{b}=1.33 \mathrm{fC}$. As shown in the left plot of Fig. 1 , diagnostics of integrated flux reveal a linear increase with $z$ for $z \geq L_{M}$. Extrapolating the linear portion back toward $z=0$ indicates a $y$-intercept equal to nearly exactly $L_{M} / 2$. The significance of this result is that for eikonal FEL simulation codes, the adiabatic matching sections at the entrance and exit to undulator segments should to lowest order be treated as being composed of a drift section and a full strength undulator section, each of length $L_{M} / 2$.

\section{Emission by a Periodic Series of Identical Ultrashort Pulses}

In order to compare the predicted results from full E\&M simulation in the boosted frame with that of a standard eikonal code, we performed a simulation of a very low current $\left(\left\langle I_{b}\right\rangle=1 \mathrm{~A}\right)$, high energy $\left(\gamma_{0}=354\right)$ long pulse $\left(l_{b}=10 \mathrm{~mm} \gg l_{\text {slip }}=2.4 \mathrm{~mm}\right)$ composed of a periodic series of ultrashort pulses, each with duration of one-eighth the resonant wavelength of $200 \mathrm{~nm}$. The energy spread and normalized emittance were sufficiently low (values of 0 and $0.1 \mathrm{~mm}$-mrad respectively) that essentially no debunching occurred over the duration of the undulator $\left(L_{u}=0.3 \mathrm{~m}, \lambda_{u}=25 \mathrm{~mm}, a_{w}=1.0\right)$. The right graph of Fig. 1 shows the radiation flux through a transverse plane $50 \mathrm{~mm}$ downstream of the undulator; the rapid oscillations correspond to half the radiation period as expected. The average power of $240 \mathrm{~W}$ measured over the interior portion of the radiation pulse (where slippage effects would disappear) is very close to the $280 \mathrm{~W}$ value for 

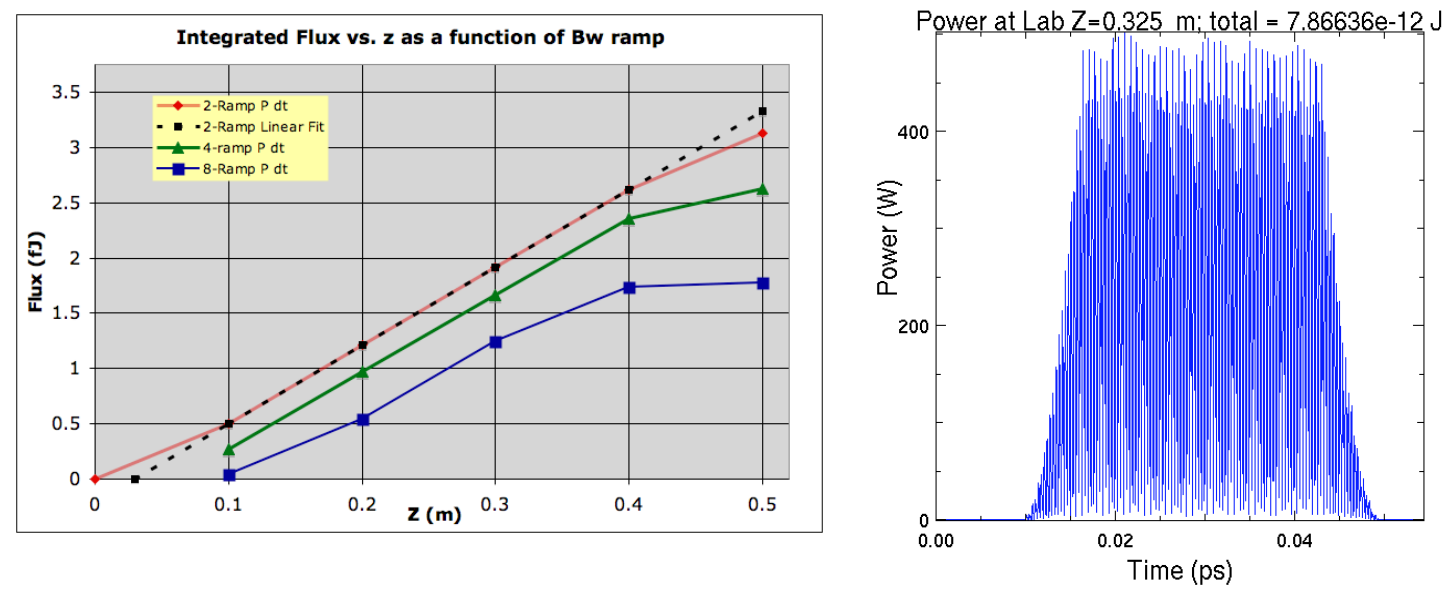

FIGURE 1. (a) WARP results for integrated 200-nm radiation flux (in the lab frame) through a transverse plane vs. $z$ for various lengths matching ramps in $B_{\perp}(z)$. From top to bottom, the ramp lengths $L_{M}$ were 5, 10, and $20 \mathrm{~mm}$ respectively with $\lambda_{u}=25 \mathrm{~mm}$. (b) Lab-frame $P(t)$ at a plane $2.5 \mathrm{~cm}$ downstream of the undulator exit for a $200-\mathrm{nm}$ FEL with 50 ultrashort microbunches. The time-averaged power over the central pulse is $240 \mathrm{~W}$. A time-steady, slab-mode GINGER run with identical e-beam parameters predicts 280 W. Please see text for more details concerning FEL parameters used to produce both figures.

a time-steady, pre-bunched pulse predicted by the eikonal GINGER code [3] (run in slab mode geometry with the same e-beam and undulator parameters taking into account the matching ramp length correction described above).

\section{Emission by a Long Pulse with Sub-harmonic Bunching}

There is great current interest among many FEL groups in exploiting modulatorradiator configurations where an e-beam is first strongly bunched by an external laser at a long wavelength $\lambda_{M}$ in the modulator and then radiates at a much shorter wavelength in the radiator, whose period and magnetic strength are tuned such that its resonant wavelength $\lambda_{R}$ is a harmonic of $\lambda_{M}$. We studied such a configuration in the boosted frame by examining emission in a "radiator" undulator from an e-beam strongly pre-bunched at $\lambda_{M}=6 \lambda_{R}$. Here again we used a very low current to minimize any complications due to gain. As expected from numerous studies using eikonal codes, on axis there is strong emission at $\lambda_{R}$ However, due to the full E\&M code being able to study emission at wavelengths much longer and shorter than $\lambda_{R}$, we can also examine sideband emission whose separation (in wavenumber) from $\lambda_{R}$ are harmonics of $\lambda_{M}$. We find that such nonresonant sideband emission does exist but, unlike radiation at the resonant wavelength for a prebunched beam, it does not grow quadratically with $z$. 


\section{HIGH GAIN SINGLE PASS AMPLIFIER SIMULATION}

In addition to the pre-bunched, ultra-low current beam simulations discussed above, we also performed a simulation of a high gain FEL amplifier. The critical e- and undulator parameters were $I_{B}=1 \mathrm{kA}$ (for each of the $e^{-}$and $e^{+}$beams), $\gamma_{0}=353.6, \varepsilon_{N}=1 \mathrm{~mm}$ $\mathrm{mrad}, \sigma_{E}=0, \lambda_{R}=200 \mathrm{~nm}, a_{w}=1.0, \lambda_{u}=25 \mathrm{~mm}$, and $N_{u}=40$. The input seed laser had $P_{0}=50 \mathrm{MW}$, and a downstream transverse waist size $\omega_{0}=250 \mu \mathrm{m}$. The effective ebeam size in the (non-resolved) $x$-dimension was $250 \mu \mathrm{m}$. From standard FEL theory, one expects a power gain length of about $0.25 \mathrm{~m}$.

The WARP simulation showed a total gain $P_{F} / P_{0}$ of approximately 5.0 in the central region of the pulse where slippage effects should not apply. A time-steady, slab-mode GINGER simulation with $L_{u}$ reduced to $0.95 \mathrm{~m}$ to take into account the adiabatic match sections showed a net gain of 5.6. On the whole, this is good quantitative agreement given the strong exponential gain for these parameters and the uncertainty in getting exactly equivalent conditions in the e-beam match between the two simulations.

\section{CONCLUSIONS}

In this paper we have discussed the application of the boosted-frame formalism of Vay [1] to short wavelength FEL simulations. The results indicate that performing electromagnetic simulation in the ponderomotive frame saves orders of magnitude in CPU time over doing the same problem in the lab frame without any obvious loss of significant physics. However, for the great bulk of FEL problems of interest at sub-IR wavelengths, despite the speed-up relative to lab frame E\&M simulation, the boosted frame approach is still orders of magnitude slower than the eikonal method. Consequently, we expect the eikonal method codes to still be used for the great bulk of FEL simulation. Nonetheless, in some instances, the boosted frame approach allows examination of effects such as matching ramps and sub-harmonic bunching that are difficult or impossible to study with eikonal codes. Further improvements (e.g., multiundulator-period averaging) may further increase the efficiency of the boosted frame method.

\section{ACKNOWLEDGMENTS}

We thank M. Zolotorev and D. Grote for helpful discussions. This work was supported under the auspices of the Office of Science, U.S. DOE under Contract No. DE-AC0205CH11231. Computational resources were provided in part by the SciDAC program and the National Energy Research Scientific Computing Center.

\section{REFERENCES}

1. J.-L Vay Phys. Rev. Lett. 98, 130405 (2007)

2. D.P. Grote, A. Friedman, J.-L. Vay, and I. Haber, AIP Conf. Proc., 749, 55 (2005).

3. W. M. Fawley, LBNL Tech. Rpt. LBNL-49625-Rev. 1 (2004); also SLAC Rpt. LCLS-TN-04-3. 


\section{DISCLAIMER}

This document was prepared as an account of work sponsored by the United States Government. While this document is believed to contain correct information, neither the United States Government nor any agency thereof, nor The Regents of the University of California, nor any of their employees, their spouses, partners, children, pets, or other associates, makes any warranty, express or implied, or assumes any legal responsibility for the accuracy, completeness, or usefulness of any information, apparatus, product, or process disclosed, or represents that its use would not infringe privately owned rights. Reference herein to any specific commercial product, process, or service by its trade name, trademark, manufacturer, or otherwise, does not necessarily constitute or imply its endorsement, recommendation, or favoring by the United States Government or any agency thereof, or The Regents of the University of California. The views and opinions of authors expressed herein do not necessarily state or reflect those of the United States Government or any agency thereof or The Regents of the University of California. 